\title{
SOME FIXED POINT THEOREMS FOR GENERALIZED $\alpha$-GERAGHTY CONTRACTION TYPE MAPPINGS IN $B$-METRIC SPACES AND SOME APPLICATIONS TO THE NONLINEAR INTEGRAL EQUATION
}

\author{
Nguyen Trung Hieu and Le Thi Chac
}

\begin{abstract}
The aim of this paper is to introduce the notion of a generalized $\alpha$-Geraghty contraction type mapping in $b$-metric spaces and state the existence and uniqueness of a fixed point for this mapping. These results are generalizations of certain main results in [D. Dukić, Z. Kadelburg, and S. Radenović, Fixed points of Geraghty-type mappings in various generalized metric spaces, Abstr. Appl. Anal. 2011 (2011), 13 pages] and [O. Popescu, Some new fixed point theorems for $\alpha$-Geraghty contraction type maps in metric spaces, Fixed Point Theory Appl. 2014:190 (2014), 1 - 12]. Some examples are given to illustrate the obtained results and to show that these results are proper extensions of the existing ones. Then we apply the obtained theorem to study the existence of solutions to the nonlinear integral equation.
\end{abstract}

Keywords: Fixed point; Generalized $\alpha$-Geraghty contraction type; $b$-metric space

\section{Introduction}

The Banach contraction principle is a useful tool in the study of many branches of mathematics and mathematical sciences. This principle was improved, generalized and extended in various ways and many fixed point results were obtained. One of the interesting generalizations of this basic principle was given by Geraghty [15] in 1973 by considering an auxiliary function. After that, Geraghty's result was generalized and many fixed point results were stated in many ways $[4,9,10,16,21]$. In particular, Popescu [26] generalized the obtained results in [10] by using the concept of triangular $\alpha$-orbital admissible mappings and studied other conditions to prove the existence and uniqueness of a fixed point of $\alpha$-Geraghty contraction type mappings in complete metric spaces. In 2016, Arshad et al. [2] introduced a generalized contraction by using the concept of triangular $\alpha$-orbital admissible mappings and stated some fixed point results in the setting of the Branciari metric space.

Received December 14., 2016.; Accepted February 18., 2017.

2010 Mathematics Subject Classification. Primary 47H10, 54H25; Secondary 54D99, 54E99 
In recent times, there were some new approaches to the generalization of the Banach contraction principle on complete metric spaces. In 2004, Ran and Reurings [27] stated a generalization of Banach contraction principle by using a partial order on a metric space. In 2008, Suzuki [30] proved a generalization of Banach contraction principle by using a contraction condition depending on a non-increasing function $\theta:[0,1) \longrightarrow\left[\frac{1}{2}, 1\right]$. In 2015, Kumam et al. [23] introduced a new generalized quasi-contraction by adding four new values $d\left(T^{2} x, x\right), d\left(T^{2} x, T x\right), d\left(T^{2} x, T y\right), d\left(T^{2} x, y\right)$ to a quasi-contraction condition. The authors also stated a unique fixed point theorem which is the generalization of Cirić fixed point theorem in [11].

Another way to generalize Banach contraction principle, appealing to many authors, was replacing the given metric space by some generalized metric space and stating analogues of fixed point theorem on metric spaces. In this way, there were many generalizations of a metric space and many fixed point theorems on generalized metric spaces were stated [5]. Note that $b$-metric is a generalization of a metric that was introduced by Czerwik in [7] and then extensively used by Czerwik in $[12,13]$. The first important difference between a metric and a $b$-metric is that the $b$-metric need not be a continuous function in its two variables, see [22, Example 13]. This led to many fixed point theorems on $b$-metric spaces being stated, so the readers may refer to $[1,3,6,8,17,18,19,20,24,25,28,29]$ and references therein.

In 2011, Dukić et al. [14] generalized the class of functions $\mathcal{F}$ to the class of functions $\mathcal{F}_{s}$ for some $s \geq 1$. By using the function $\beta \in \mathcal{F}_{s}$, the authors stated the existence and uniqueness of a fixed point for Geraghty contraction type mapping in $b$-metric spaces.

The aim of this paper is to introduce the notion of a generalized $\alpha$-Geraghty contraction type mapping in $b$-metric spaces by adding four terms $d\left(T^{2} x, T x\right)$, $\frac{d\left(T^{2} x, x\right)+d\left(T^{2} x, T y\right)}{2 s}, d\left(T^{2} x, y\right), d\left(T^{2} x, T y\right)$ to the contractive condition in [26, Definition 4] and state the existence and uniqueness of a fixed point for this mapping. Some examples are given to illustrate the obtained results and to show that these results are proper extensions of the existing ones. Then we apply the obtained theorem to study the existence of solutions to the nonlinear integral equation.

\section{Preliminaries}

The following interesting result was given by Geraghty [15] in 1973 by considering an auxiliary function.

Theorem 2.1. [15] Let $(X, d)$ be a complete metric spaces and $T: X \longrightarrow X$ be a mapping. Suppose that there exists $\beta \in \mathcal{F}$ such that for all $x, y \in X$,

$$
d(T x, T y) \leqslant \beta(d(x, y)) d(x, y),
$$

where $\mathcal{F}$ is the family of all functions $\beta:[0, \infty) \longrightarrow[0,1)$ such that

$$
\lim _{n \rightarrow \infty} \beta\left(t_{n}\right)=1 \text { implies } \lim _{n \rightarrow \infty} t_{n}=0 .
$$


Then $T$ has a unique fixed point $z \in X$ and $\left\{T^{n} x\right\}$ converges to $z$ for each $x \in X$.

In 2014, Popescu [26] studied the existence and uniqueness of a fixed point of $\alpha$-Geraghty contraction type mappings in complete metric space.

Definition 2.1. [26] Let $T: X \longrightarrow X$ be a mapping and $\alpha: X \times X \longrightarrow \mathbb{R}$ be a function. Then $T$ is called $\alpha$-orbital admissible if $\alpha(x, T x) \geq 1$ implies $\alpha\left(T x, T^{2} x\right) \geq 1$ for all $x \in X$.

Definition 2.2. [26] Let $T: X \longrightarrow X$ be a mapping and $\alpha: X \times X \longrightarrow \mathbb{R}$ be a function. Then $T$ is called triangular $\alpha$-orbital admissible if

1. $T$ is $\alpha$-orbital admissible.

2. $\alpha(x, y) \geq 1$ and $\alpha(y, T y) \geq 1$ imply $\alpha(x, T y) \geq 1$ for all $x, y \in X$.

Definition 2.3. $[26]$ Let $(X, d)$ be a metric space, $\alpha: X \times X \longrightarrow \mathbb{R}$ be a function and $T: X \longrightarrow X$ be a mapping. Then $T$ is called a generalized $\alpha$-Geraghty contraction type mapping if there exists $\beta \in \mathcal{F}$ such that for all $x, y \in X$,

$$
\alpha(x, y) d(T x, T y) \leqslant \beta\left(M_{T}(x, y)\right) M_{T}(x, y),
$$

where

$$
M_{T}(x, y)=\max \left\{d(x, y), d(x, T x), d(y, T y), \frac{d(x, T y)+d(y, T x)}{2}\right\} .
$$

The notion of a $b$-metric space and some notions on $b$-metric spaces was introduced as follows.

Definition 2.4. [13] Let $X$ be a non-empty set and $d: X \times X \longrightarrow[0, \infty)$ be a function such that for all $x, y, z \in X$ and some $s \geq 1$,

1. $d(x, y)=0$ if and only if $x=y$.

2. $d(x, y)=d(y, x)$.

3. $d(x, y) \leq s(d(x, z)+d(z, y))$.

Then $d$ is called a $b$-metric on $X$ and $(X, d, s)$ is called a $b$-metric space.

Definition 2.5. [13] Let $(X, d, s)$ be a $b$-metric space. Then

1. A sequence $\left\{x_{n}\right\}$ is called convergent to $x$, written $\lim _{n \rightarrow \infty} x_{n}=x$, if $\lim _{n \rightarrow \infty} d\left(x_{n}, x\right)=0$.

2. A sequence $\left\{x_{n}\right\}$ is called Cauchy in $X$ if $\lim _{n, m \rightarrow \infty} d\left(x_{n}, x_{m}\right)=0$. 
3. $(X, d, s)$ is called complete if each Cauchy sequence is a convergent sequence.

Aghajani et al. [1] proved the following simple lemma about the convergent sequence in $b$-metric spaces.

Lemma 2.1. [1] Let $(X, d, s)$ be a b-metric space and $\lim _{n \rightarrow \infty} x_{n}=x, \lim _{n \rightarrow \infty} y_{n}=y$. Then

1. $\frac{1}{s^{2}} d(x, y) \leq \liminf _{n \rightarrow \infty} d\left(x_{n}, y_{n}\right) \leq \limsup _{n \rightarrow \infty} d\left(x_{n}, y_{n}\right) \leq s^{2} d(x, y)$. In particular, if $x=y$, then $\lim _{n \rightarrow \infty} d\left(x_{n}, y_{n}\right)=0$.

2. For each $z \in X, \frac{1}{s} d(x, z) \leq \liminf _{n \rightarrow \infty} d\left(x_{n}, z\right) \leq \limsup _{n \rightarrow \infty} d\left(x_{n}, z\right) \leq \operatorname{sd}(x, z)$.

Dukić et al. [14] stated the existence and uniqueness of a fixed point for Geraghty contraction type mapping in $b$-metric spaces by using the function $\beta \in \mathcal{F}_{s}$.

Lemma 2.2. [26] Let $T: X \longrightarrow X$ be a triangular $\alpha$-orbital admissible mapping. Assume that there exists $x_{1} \in X$ such that $\alpha\left(x_{1}, T x_{1}\right) \geqslant 1$. Define a sequence $\left\{x_{n}\right\}$ by $x_{n+1}=T x_{n}$ for all $n \geq 1$. Then $\alpha\left(x_{n}, x_{m}\right) \geqslant 1$ for all $m>n \geq 1$.

Theorem 2.2. [14] Let $(X, d, s)$ be a complete $b$-metric space and $T: X \longrightarrow X$ be a mapping. Suppose that there exists $\beta \in \mathcal{F}_{s}$ such that for all $x, y \in X$,

$$
d(T x, T y) \leqslant \beta(d(x, y)) d(x, y)
$$

where $\mathcal{F}_{s}$ is the family of all functions $\beta:[0, \infty) \longrightarrow\left[0, \frac{1}{s}\right)$ which satisfies the following condition: $\lim _{n \rightarrow \infty} \beta\left(t_{n}\right)=\frac{1}{s}$ implies $\lim _{n \rightarrow \infty} t_{n}=0$. Then $T$ has a unique fixed point $z \in X$ and $\left\{T^{n} x\right\}$ converges to $z$ for each $x \in X$.

\section{Main results}

First, by using the function $\beta \in \mathcal{F}_{s}$ and adding four terms $\frac{d\left(T^{2} x, x\right)+d\left(T^{2} x, T y\right)}{2 s}$, $d\left(T^{2} x, T x\right), d\left(T^{2} x, y\right), d\left(T^{2} x, T y\right)$ to the generalized $\alpha$-Geraghty contraction condition in metric spaces, we introduce the notion of a generalized $\alpha$-Geraghty contraction type mapping in $b$-metric spaces.

Definition 3.1. Let $(X, d, s)$ be a $b$-metric space, $\alpha: X \times X \longrightarrow \mathbb{R}$ be a function and $T: X \longrightarrow X$ be a mapping. Then $T$ is called a generalized $\alpha$-Geraghty contraction type if there exists $\beta \in \mathcal{F}_{s}$ such that for all $x, y \in X$,

$$
s \alpha(x, y) d(T x, T y) \leqslant \beta\left(C_{s}(x, y)\right) C_{s}(x, y)
$$


where

$$
\begin{aligned}
C_{s}(x, y)= & \max \left\{d(x, y), d(x, T x), d(y, T y), \frac{d(x, T y)+d(y, T x)}{2 s}\right. \\
& \left.\frac{d\left(T^{2} x, x\right)+d\left(T^{2} x, T y\right)}{2 s}, d\left(T^{2} x, T x\right), d\left(T^{2} x, T y\right), d\left(T^{2} x, y\right)\right\}
\end{aligned}
$$

The following theorem is a sufficient condition for the existence of the fixed point for a generalized $\alpha$-Geraghty contraction type mapping in $b$-metric spaces.

Theorem 3.1. Let $(X, d, s)$ be a complete b-metric space, $\alpha: X \times X \longrightarrow \mathbb{R}$ be a function and let $T: X \longrightarrow X$ be a mapping such that

1. $T$ is a generalized $\alpha$-Geraghty contraction type mapping.

2. $T$ is a triangular $\alpha$-orbital admissible mapping.

3. There exists $x_{1} \in X$ such that $\alpha\left(x_{1}, T x_{1}\right) \geqslant 1$.

4. $T$ is continuous.

Then $T$ has a fixed point $z \in X$ and $\left\{T^{n} x_{1}\right\}$ converges to $z$.

Proof. Let $x_{1} \in X$ such that $\alpha\left(x_{1}, T x_{1}\right) \geqslant 1$, we construct a sequence $\left\{x_{n}\right\}$ by $x_{n+1}=T x_{n}$ for $n \geq 1$. By using Lemma 2.2, we have $\alpha\left(x_{n}, x_{n+1}\right) \geqslant 1$ for all $n \geqslant 1$. Since $T$ is a generalized $\alpha$-Geraghty contraction type mapping, we have

$$
\begin{aligned}
s d\left(x_{n+1}, x_{n+2}\right) & =s d\left(T x_{n}, T x_{n+1}\right) \\
& \leqslant s \alpha\left(x_{n}, x_{n+1}\right) d\left(T x_{n}, T x_{n+1}\right) \\
& \leqslant \beta\left(C_{s}\left(x_{n}, x_{n+1}\right)\right) C_{s}\left(x_{n}, x_{n+1}\right) .
\end{aligned}
$$

We also have

$$
\begin{aligned}
& \max \left\{d\left(x_{n}, x_{n+1}\right), d\left(x_{n+1}, x_{n+2}\right)\right\} \\
\leq & C_{s}\left(x_{n}, x_{n+1}\right) \\
= & \max \left\{d\left(x_{n}, x_{n+1}\right), d\left(x_{n}, T x_{n}\right), d\left(x_{n+1}, T x_{n+1}\right)\right. \\
& \frac{d\left(x_{n}, T x_{n+1}\right)+d\left(x_{n+1}, T x_{n}\right)}{2 s}, \frac{d\left(T^{2} x_{n}, x_{n}\right)+d\left(T^{2} x_{n}, T x_{n+1}\right)}{2 s}, \\
& \left.d\left(T^{2} x_{n}, T x_{n}\right), d\left(T^{2} x_{n}, T x_{n+1}\right), d\left(T^{2} x_{n}, x_{n+1}\right)\right\}
\end{aligned}
$$




$$
\begin{aligned}
= & \max \left\{d\left(x_{n}, x_{n+1}\right), d\left(x_{n}, x_{n+1}\right), d\left(x_{n+1}, x_{n+2}\right),\right. \\
& \frac{d\left(x_{n}, x_{n+2}\right)+d\left(x_{n+1}, x_{n+1}\right)}{2 s}, \frac{d\left(x_{n+2}, x_{n}\right)+d\left(x_{n+2}, x_{n+2}\right)}{2 s}, \\
& \left.d\left(x_{n+2}, x_{n+1}\right), d\left(x_{n+2}, x_{n+2}\right), d\left(x_{n+2}, x_{n+1}\right)\right\} \\
= & \max \left\{d\left(x_{n}, x_{n+1}\right), d\left(x_{n+1}, x_{n+2}\right), \frac{d\left(x_{n}, x_{n+2}\right)}{2 s}\right\} \\
\leq & \max \left\{d\left(x_{n}, x_{n+1}\right), d\left(x_{n+1}, x_{n+2}\right), \frac{d\left(x_{n}, x_{n+1}\right)+d\left(x_{n+1}, x_{n+2}\right)}{2}\right\} \\
= & \max \left\{d\left(x_{n}, x_{n+1}\right), d\left(x_{n+1}, x_{n+2}\right)\right\} .
\end{aligned}
$$

This implies that

$$
C_{s}\left(x_{n}, x_{n+1}\right)=\max \left\{d\left(x_{n}, x_{n+1}\right), d\left(x_{n+1}, x_{n+2}\right)\right\} .
$$

From (3.2) and (3.3), we have

$$
\begin{aligned}
& s d\left(x_{n+1}, x_{n+2}\right) \\
(3.4) \leqslant & \beta\left(\max \left\{d\left(x_{n}, x_{n+1}\right), d\left(x_{n+1}, x_{n+2}\right)\right\}\right) \max \left\{d\left(x_{n}, x_{n+1}\right), d\left(x_{n+1}, x_{n+2}\right)\right\} .
\end{aligned}
$$

If there exists $n \geqslant 1$ such that $\max \left\{d\left(x_{n}, x_{n+1}\right), d\left(x_{n+1}, x_{n+2}\right)\right\}=d\left(x_{n+1}, x_{n+2}\right)$, then (3.4) becomes

$$
\begin{aligned}
s d\left(x_{n+1}, x_{n+2}\right) & \leqslant \beta\left(d\left(x_{n+1}, x_{n+2}\right)\right) d\left(x_{n+1}, x_{n+2}\right) \\
& <\frac{1}{s} d\left(x_{n+1}, x_{n+2}\right) .
\end{aligned}
$$

This implies that

$$
\frac{s^{2}-1}{s} d\left(x_{n+1}, x_{n+2}\right)<0 .
$$

This leads to a contradiction with $s \geqslant 1$. Therefore, $\max \left\{d\left(x_{n}, x_{n+1}\right), d\left(x_{n+1}, x_{n+2}\right)\right\}=$ $d\left(x_{n}, x_{n+1}\right)$ for all $n \geqslant 1$. Then $(3.4)$ becomes

$$
\begin{aligned}
s d\left(x_{n+1}, x_{n+2}\right) & \leqslant \beta\left(d\left(x_{n}, x_{n+1}\right)\right) d\left(x_{n}, x_{n+1}\right) \\
& <\frac{1}{s} d\left(x_{n}, x_{n+1}\right) \\
& \leq s d\left(x_{n}, x_{n+1}\right) .
\end{aligned}
$$

It follows that $\left\{d\left(x_{n}, x_{n+1}\right)\right\}$ is a non-decreasing sequence of non-negative real numbers. Therefore, there exists $r \geq 0$ such that $\lim _{n \rightarrow \infty} d\left(x_{n}, x_{n+1}\right)=r$. We will show that $r=0$. On the contrary, suppose that $r>0$. From $(3.5)$, we obtain

$$
\begin{aligned}
\frac{1}{s} d\left(x_{n+1}, x_{n+2}\right) & \leqslant \beta\left(d\left(x_{n}, x_{n+1}\right)\right) d\left(x_{n}, x_{n+1}\right) \\
& <\frac{1}{s} d\left(x_{n}, x_{n+1}\right) .
\end{aligned}
$$


Letting $n \rightarrow \infty$ in (3.6), we get

$$
\frac{1}{s} r \leqslant r \lim _{n \rightarrow \infty} \beta\left(d\left(x_{n}, x_{n+1}\right)\right) \leq \frac{1}{s} r .
$$

This implies that $\lim _{n \rightarrow \infty} \beta\left(d\left(x_{n}, x_{n+1}\right)\right)=\frac{1}{s}$. Since $\beta \in \mathcal{F}_{s}$, we obtain

$$
\lim _{n \rightarrow \infty} d\left(x_{n}, x_{n+1}\right)=0,
$$

which contradicts $r=0$. Therefore, we conclude that

$$
\lim _{n \rightarrow \infty} d\left(x_{n}, x_{n+1}\right)=0 .
$$

Next, we will prove that $\left\{x_{n}\right\}$ is a Cauchy sequence. On the contrary, suppose that $\left\{x_{n}\right\}$ is not a Cauchy sequence. Then there exists $\varepsilon>0$ for which we can find two subsequence $\left\{x_{n(k)}\right\}$ and $\left\{x_{m(k)}\right\}$ of $\left\{x_{n}\right\}$ such that $m(k)$ is the smallest index satisfying $m(k)>n(k)>k \geq 1$ and

$$
d\left(x_{n(k)}, x_{m(k)}\right) \geqslant \varepsilon .
$$

This implies that

$$
d\left(x_{n(k)}, x_{m(k)-1}\right)<\varepsilon .
$$

Then, from (3.8) and (3.9), we get

$$
\begin{aligned}
\varepsilon & \leqslant d\left(x_{n(k)}, x_{m(k)}\right) \\
& \leqslant s d\left(x_{n(k)}, x_{m(k)-1}\right)+s d\left(x_{m(k)}, x_{m(k)-1}\right) \\
& <\varepsilon s+s d\left(x_{m(k)}, x_{m(k)-1}\right) .
\end{aligned}
$$

Letting $k \rightarrow \infty$ in (3.10) and using (3.7), we have

$$
\varepsilon \leqslant \liminf _{k \rightarrow \infty} d\left(x_{n(k)}, x_{m(k)}\right) \leqslant \limsup _{k \rightarrow \infty} d\left(x_{n(k)}, x_{m(k)}\right) \leqslant \varepsilon s .
$$

From (3.8), we again have

$$
\begin{aligned}
\varepsilon & \leqslant d\left(x_{m(k)}, x_{n(k)}\right) \\
& \leqslant s d\left(x_{m(k)}, x_{m(k)-1}\right)+s d\left(x_{m(k)-1}, x_{n(k)}\right) \\
& \leqslant s d\left(x_{m(k)}, x_{m(k)-1}\right)+s^{2} d\left(x_{m(k)-1}, x_{n(k)-1}\right)+s^{2} d\left(x_{n(k)-1}, x_{n(k)}\right) .
\end{aligned}
$$

Letting $k \rightarrow \infty$ in (3.12) and using (3.7), we have

$$
\frac{\varepsilon}{s^{2}} \leqslant \liminf _{k \rightarrow \infty} d\left(x_{n(k)-1}, x_{m(k)-1}\right) \leqslant \limsup _{k \rightarrow \infty} d\left(x_{n(k)-1}, x_{m(k)-1}\right) .
$$

From (3.9), we also have

$$
\begin{aligned}
d\left(x_{n(k)-1}, x_{m(k)-1}\right) & \leqslant s d\left(x_{n(k)-1}, x_{n(k)}\right)+s d\left(x_{n(k)}, x_{m(k)-1}\right) \\
& <s d\left(x_{n(k)-1}, x_{n(k)}\right)+s \varepsilon .
\end{aligned}
$$


Letting $k \rightarrow \infty$ in (3.14) and using (3.7), we get

$$
\liminf _{k \rightarrow \infty} d\left(x_{n(k)-1}, x_{m(k)-1}\right) \leqslant \limsup _{k \rightarrow \infty} d\left(x_{n(k)-1}, x_{m(k)-1}\right) \leqslant \varepsilon s .
$$

Combining (3.13) with (3.15), we obtain

$$
\frac{\varepsilon}{s^{2}} \leqslant \liminf _{k \rightarrow \infty} d\left(x_{n(k)-1}, x_{m(k)-1}\right) \leqslant \limsup _{k \rightarrow \infty} d\left(x_{n(k)-1}, x_{m(k)-1}\right) \leqslant \varepsilon s .
$$

From (3.8), we also have

$$
\varepsilon \leqslant d\left(x_{m(k)}, x_{n(k)}\right) \leqslant s d\left(x_{m(k)}, x_{m(k)-1}\right)+s d\left(x_{m(k)-1}, x_{n(k)}\right) .
$$

Letting $k \rightarrow \infty$ in (3.17) and using (3.7), we have

$$
\frac{\varepsilon}{s} \leqslant \liminf _{k \rightarrow \infty} d\left(x_{m(k)-1}, x_{n(k)}\right) \leqslant \limsup _{k \rightarrow \infty} d\left(x_{m(k)-1}, x_{n(k)}\right) \leqslant \varepsilon s .
$$

We also have

$$
\varepsilon \leqslant d\left(x_{m(k)}, x_{n(k)}\right) \leqslant s d\left(x_{m(k)}, x_{n(k)-1}\right)+s d\left(x_{n(k)-1}, x_{n(k)}\right),
$$

Letting $k \rightarrow \infty$ in (3.19), (3.20) and using (3.7), (3.16), we get

$$
\frac{\varepsilon}{s} \leqslant \liminf _{k \rightarrow \infty} d\left(x_{n(k)-1}, x_{m(k)}\right) \leqslant \limsup _{k \rightarrow \infty} d\left(x_{n(k)-1}, x_{m(k)}\right) \leqslant \varepsilon s^{2} .
$$

We again have

$$
\varepsilon \leq d\left(x_{n(k)}, x_{m(k)}\right) \leq s d\left(x_{n(k)}, x_{n(k)+1}\right)+s d\left(x_{n(k)+1}, x_{m(k)}\right),
$$

$$
d\left(x_{n(k)+1}, x_{m(k)}\right) \leq s d\left(x_{n(k)+1}, x_{n(k)}\right)+s d\left(x_{n(k)}, x_{m(k)}\right) .
$$

Letting $k \rightarrow \infty$ in (3.22), (3.23) and using (3.7), (3.11), we obtain

$$
\frac{\varepsilon}{s} \leqslant \liminf _{k \rightarrow \infty} d\left(x_{n(k)+1}, x_{m(k)}\right) \leqslant \limsup _{k \rightarrow \infty} d\left(x_{n(k)+1}, x_{m(k)}\right) \leqslant \varepsilon s^{2} .
$$

We also have

$$
\begin{aligned}
& d\left(x_{m(k)-1}, x_{n(k)}\right) \leq s d\left(x_{m(k)-1}, x_{n(k)+1}\right)+s d\left(x_{n(k)+1}, x_{n(k)}\right), \\
& d\left(x_{n(k)+1}, x_{m(k)-1}\right) \leq s d\left(x_{n(k)+1}, x_{n(k)}\right)+s d\left(x_{n(k)}, x_{m(k)-1}\right) .
\end{aligned}
$$

Letting $k \rightarrow \infty$ in (3.25), (3.26) and using (3.7), (3.9), (3.18), we have

$$
\frac{\varepsilon}{s^{2}} \leqslant \liminf _{k \rightarrow \infty} d\left(x_{n(k)+1}, x_{m(k)-1}\right) \leqslant \limsup _{k \rightarrow \infty} d\left(x_{n(k)+1}, x_{m(k)-1}\right) \leqslant \varepsilon s .
$$


Since $m(k)-1>n(k)-1$, we get $\alpha\left(x_{n(k)-1}, x_{m(k)-1}\right) \geqslant 1$ by Lemma 2.2 . Then, from (3.1), we have

$$
\begin{aligned}
s d\left(x_{n(k)}, x_{m(k)}\right) & =s d\left(T x_{n(k)-1}, T x_{m(k)-1}\right) \\
& \leqslant s \alpha\left(x_{n(k)-1}, x_{m(k)-1}\right) d\left(T x_{n(k)-1}, T x_{m(k)-1}\right) \\
& \leqslant \beta\left(C_{s}\left(x_{n(k)-1}, x_{m(k)-1}\right)\right) C_{s}\left(x_{n(k)-1}, x_{m(k)-1}\right),
\end{aligned}
$$

where

$$
\begin{aligned}
& C_{s}\left(x_{n(k)-1}, x_{m(k)-1}\right) \\
= & \max \left\{d\left(x_{n(k)-1}, x_{m(k)-1}\right), d\left(x_{n(k)-1}, T x_{n(k)-1}\right), d\left(x_{m(k)-1}, T x_{m(k)-1}\right),\right. \\
& \frac{d\left(x_{n(k)-1}, T x_{m(k)-1}\right)+d\left(x_{m(k)-1}, T x_{n(k)-1}\right)}{2 s}, \\
& \frac{d\left(T^{2} x_{n(k)-1}, x_{n(k)-1}\right)+d\left(T^{2} x_{n(k)-1}, T x_{m(k)-1}\right)}{2 s}, \\
= & \max \left\{d\left(T^{2} x_{n(k)-1}, T x_{n(k)-1}\right), d\left(T^{2} x_{n(k)-1}, T x_{m(k)-1}\right), d\left(T^{2} x_{n(k)-1}, x_{m(k)-1}\right)\right\} \\
& \frac{d\left(x_{n(k)-1}, x_{m(k)}\right)+d\left(x_{m(k)-1}, x_{n(k)}\right)}{2 s}, \frac{d\left(x_{n(k)+1}, x_{n(k)-1}\right)+d\left(x_{n(k)+1}, x_{m(k)}\right)}{2 s}, \\
(3.29) & \left.\quad\left(x_{n(k)+1}, x_{n(k)}\right), d\left(x_{n(k)+1}, x_{m(k)}\right), d\left(x_{n(k)+1}, x_{m(k)-1}\right)\right\} .
\end{aligned}
$$

Letting $k \rightarrow \infty$ in (3.29) and using (3.7), (3.16), (3.18), (3.21), (3.24) and (3.27), we have

$$
\begin{aligned}
\frac{\varepsilon}{s^{2}} & =\max \left\{\frac{\varepsilon}{s^{2}}, 0,0, \frac{\frac{\varepsilon}{s}+\frac{\varepsilon}{s}}{2 s}, \frac{0+\frac{\varepsilon}{s}}{2 s}, 0, \frac{\varepsilon}{s}, \frac{\varepsilon}{s^{2}}\right\} \\
& \leq \liminf _{k \rightarrow \infty} C_{s}\left(x_{n(k)-1}, x_{m(k)-1}\right) \\
& \leq \limsup _{k \rightarrow \infty} C_{s}\left(x_{n(k)-1}, x_{m(k)-1}\right) \\
& \leq \max \left\{\varepsilon s, 0,0, \frac{\varepsilon s^{2}+\varepsilon s^{2}}{2 s}, \frac{0+\varepsilon s^{2}}{2 s}, 0, \varepsilon s^{2}, \varepsilon s\right\} \\
& =\varepsilon s^{2} .
\end{aligned}
$$

Letting $k \rightarrow \infty$ in (3.28) and using (3.30), we have

$$
\begin{aligned}
s \varepsilon & \leqslant \limsup _{n \rightarrow \infty} \beta\left(C_{s}\left(x_{n}, x_{n+1}\right)\right) \limsup _{n \rightarrow \infty} C_{s}\left(x_{n}, x_{n+1}\right) \\
& \leq \varepsilon s^{2} \limsup _{n \rightarrow \infty} \beta\left(C_{s}\left(x_{n}, x_{n+1}\right)\right) .
\end{aligned}
$$

This implies that $\limsup _{n \rightarrow \infty} \beta\left(C_{s}\left(x_{n}, x_{n+1}\right)\right) \geq \frac{1}{s}$. Since $\beta \in \mathcal{F}_{s}$, we have

$$
\limsup _{n \rightarrow \infty} \beta\left(C_{s}\left(x_{n}, x_{n+1}\right)\right) \leq \frac{1}{s} .
$$


We conclude that

$$
\limsup _{n \rightarrow \infty} \beta\left(C_{s}\left(x_{n}, x_{n+1}\right)\right)=\frac{1}{s}
$$

Similary, we also see that

$$
\liminf _{n \rightarrow \infty} \beta\left(C_{s}\left(x_{n}, x_{n+1}\right)\right)=\frac{1}{s} .
$$

Combining (3.31) with (3.32), we conclude that $\lim _{n \rightarrow \infty} \beta\left(C_{s}\left(x_{n}, x_{n+1}\right)\right)=\frac{1}{s}$. Since $\beta \in \mathcal{F}_{s}$, we have $\lim _{n \rightarrow \infty} C_{s}\left(x_{n}, x_{n+1}\right)=0$, contrary to (3.30). Thus, $\left\{x_{n}\right\}$ is a Cauchy sequence in $(X, d, s)$. Since $(X, d, s)$ is complete, there exists $z \in X$ such that $\lim _{n \rightarrow \infty} x_{n}=z$. Since $T$ is continuous, we obtain $z=\lim _{n \rightarrow \infty} x_{n}=\lim _{n \rightarrow \infty} x_{n+1}=$ $\lim _{n \rightarrow \infty} T x_{n}=T\left(\lim _{n \rightarrow \infty} x_{n}\right)=T z$. It implies that $z$ is a fixed point of $T$. Since $\lim _{n \rightarrow \infty} x_{n+1}=z$ and $x_{n+1}=T x_{n}=T^{n} x_{1}, \lim _{n \rightarrow \infty} T^{n} x_{1}=z$.

In the following theorem, we replace the continuity of a mapping $T$ in Theorem 3.1 by another condition.

Theorem 3.2. Let $(X, d, s)$ be a complete b-metric space, $\alpha: X \times X \longrightarrow \mathbb{R}$ be a function and $T: X \longrightarrow X$ be a mapping such that

1. $T$ is a generalized $\alpha$-Geraghty contraction type mapping.

2. $T$ is a triangular $\alpha$-orbital admissible mapping.

3. There exists $x_{1} \in X$ such that $\alpha\left(x_{1}, T x_{1}\right) \geqslant 1$.

4. If $\left\{x_{n}\right\}$ is a sequence in $X$ such that $\alpha\left(x_{n}, x_{n+1}\right) \geqslant 1$ for all $n \geqslant 1$ and $\lim _{n \rightarrow \infty} x_{n}=x \in X$, then there exists a subsequence $\left\{x_{n(k)}\right\}$ of $\left\{x_{n}\right\}$ such that $\alpha\left(x_{n(k)}, x\right) \geqslant 1$ for all $k \geqslant 1$.

Then $T$ has a fixed point $z \in X$ and $\left\{T^{n} x_{1}\right\}$ converges to $z$.

Proof. Following the lines in the proof of Theorem 3.1, we conclude that the sequence $\left\{x_{n}\right\}$ by $x_{n+1}=T x_{n}$ for $n \geq 1$ converges to $z \in X$. By using hypothesis (4), we deduce that there exists a subsequence $\left\{x_{n(k)}\right\}$ of $\left\{x_{n}\right\}$ such that $\alpha\left(x_{n(k)}, z\right) \geq 1$. Since $T$ is a generalized $\alpha$-Geraghty contraction type mapping, we obtain

$$
\begin{aligned}
s d\left(x_{n(k)+1}, T z\right) & =s d\left(T x_{n(k)}, T z\right) \\
& \leqslant s \alpha\left(x_{n(k)}, z\right) d\left(T x_{n(k)}, T z\right) \\
& \leqslant \beta\left(C_{s}\left(x_{n(k)}, z\right)\right) C_{s}\left(x_{n(k)}, z\right),
\end{aligned}
$$


where

$$
\begin{aligned}
C_{s}\left(x_{n(k)}, z\right)= & \max \left\{d\left(x_{n(k)}, z\right), d\left(x_{n(k)}, T x_{n(k)}\right), d(z, T z),\right. \\
& \frac{d\left(x_{n(k)}, T z\right)+d\left(z, T x_{n(k)}\right)}{2 s}, \frac{d\left(T^{2} x_{n(k)}, x_{n(k)}\right)+d\left(T^{2} x_{n(k)}, T z\right)}{2 s}, \\
& \left.d\left(T^{2} x_{n(k)}, T x_{n(k)}\right), d\left(T^{2} x_{n(k)}, T z\right), d\left(T^{2} x_{n(k)}, z\right)\right\} \\
= & \max \left\{d\left(x_{n(k)}, z\right), d\left(x_{n(k)}, x_{n(k)+1}\right), d(z, T z),\right. \\
& \frac{d\left(x_{n(k)}, T z\right)+d\left(z, x_{n(k)+1}\right)}{2 s}, \frac{d\left(x_{n(k)+2}, x_{n(k)}\right)+d\left(x_{n(k)+2}, T z\right)}{2 s}, \\
& \left.d\left(x_{n(k)+2}, x_{n(k)+1}\right), d\left(x_{n(k)+2}, T z\right), d\left(x_{n(k)+2}, z\right)\right\} .
\end{aligned}
$$

On the contrary, suppose that $T z \neq z$. Then $d(T z, z)>0$. Letting $k \rightarrow \infty$ in (3.34) and using (3.7), Lemma 2.1, we have

$$
\frac{1}{s^{2}} d(z, T z) \leq \liminf _{n \rightarrow \infty} C_{s}\left(x_{n(k)}, z\right) \leq \limsup _{n \rightarrow \infty} C_{s}\left(x_{n(k)}, z\right) \leq s d(z, T z) .
$$

Letting $k \rightarrow \infty$ in (3.33) and using (3.35), Lemma 2.1, we get

$$
d(z, T z)=s\left(\frac{1}{s} d(z, T z)\right) \leqslant s d(z, T z) \limsup _{n \rightarrow \infty} \beta\left(C_{s}\left(x_{n(k)}, z\right)\right) .
$$

This implies that $\limsup _{n \rightarrow \infty} \beta\left(C_{s}\left(x_{n(k)}, z\right)\right) \geq \frac{1}{s}$. Since $\beta \in \mathcal{F}_{s}$, we have

$$
\limsup _{n \rightarrow \infty} \beta\left(C_{s}\left(x_{n(k)}, z\right)\right) \leq \frac{1}{s}
$$

We conclude that

$$
\limsup _{n \rightarrow \infty} \beta\left(C_{s}\left(x_{n(k)}, z\right)\right)=\frac{1}{s}
$$

Similarly, we also see that

$$
\liminf _{n \rightarrow \infty} \beta\left(C_{s}\left(x_{n(k)}, z\right)\right)=\frac{1}{s} .
$$

Combining (3.36) with (3.37), we get $\lim _{n \rightarrow \infty} \beta\left(C_{s}\left(x_{n(k)}, z\right)\right)=\frac{1}{s}$. Since $\beta \in \mathcal{F}_{s}$, we have $\lim _{n \rightarrow \infty} C_{s}\left(x_{n(k)}, z\right)=0$, which 0 (3.35). This implies that $T z=z$. Therefore, $z$ is a fixed point of $T$. Since $\lim _{n \rightarrow \infty} x_{n+1}=z$ and $x_{n+1}=T x_{n}=T^{n} x_{1}$, we get that $\lim _{n \rightarrow \infty} T^{n} x_{1}=z$.

The following theorem is a sufficient condition for the uniqueness of a fixed point for a generalized $\alpha$-Geraghty contraction type mapping in $b$-metric spaces. 
Theorem 3.3. Let $(X, d, s)$ be a complete b-metric space, $\alpha: X \times X \longrightarrow \mathbb{R}$ be a function and $T: X \longrightarrow X$ be a mapping such that

1. All the hypotheses in Theorem 3.1 or Theorem 3.2 are satisfied.

2. If $x \neq y$ are two fixed points of $T$, then there exists $v \in X$ such that $\alpha(x, v) \geq$ $1, \alpha(y, v) \geq 1$ and $\alpha(v, T v) \geq 1$.

Then $T$ has a unique fixed point.

Proof. By Theorem 3.1 and Theorem 3.2, $T$ has a fixed point. Suppose that $x$ and $y$ are two fixed points of $T$ with $x \neq y$. By using hypothesis (2), there exists $v \in X$ such that $\alpha(x, v) \geq 1, \alpha(y, v) \geq 1$ and $\alpha(v, T v) \geq 1$. Since $\alpha(x, v) \geq 1$ and $\alpha(v, T v) \geq 1$, we have $\alpha(x, T v) \geq 1$. Since $\alpha(v, T v) \geq 1$, we also have $\alpha\left(T v, T^{2} v\right) \geq$ 1. This implies that $\alpha\left(x, T^{2} v\right) \geq 1$. Continuing this process, we get $\alpha\left(x, T^{n} v\right) \geq 1$ for all $n \geq 1$. Similarly, we also see that $\alpha\left(y, T^{n} v\right) \geq 1$ for all $n \geq 1$. Since $\alpha\left(x, T^{n} v\right) \geq 1$ and $T$ is a generalized $\alpha$-Geraghty contraction type mapping, we obtain

$$
\begin{aligned}
s d\left(x, T^{n+1} v\right) & =s d\left(T x, T^{n+1} v\right) \\
& \leqslant s \alpha\left(x, T^{n} v\right) d\left(x, T^{n} v\right) \\
& \leqslant \beta\left(C_{s}\left(x, T^{n} v\right)\right) C_{s}\left(x, T^{n} v\right),
\end{aligned}
$$

where

$$
\begin{aligned}
& C_{s}\left(x, T^{n} v\right) \\
= & \max \left\{d\left(x, T^{n} v\right), d(x, T x), d\left(T^{n} v, T^{n+1} v\right), \frac{d\left(x, T^{n+1} v\right)+d\left(T^{n} v, T x\right)}{2 s},\right. \\
& \left.\frac{d\left(T^{2} x, x\right)+d\left(T^{2} x, T^{n+1} v\right)}{2 s}, d\left(T^{2} x, T x\right), d\left(T^{2} x, T^{n+1} v\right), d\left(T^{2} x, T^{n} v\right)\right\} \\
9= & \max \left\{d\left(x, T^{n} v\right), d\left(T^{n} v, T^{n+1} v\right), \frac{d\left(x, T^{n+1} v\right)+d\left(T^{n} v, x\right)}{2 s}, d\left(x, T^{n+1} v\right)\right\} .
\end{aligned}
$$

It follows from Theorem 3.1 or Theorem 3.2 where $x_{1}$ is replaced by $v$ that there exists $z \in X$ such that $\lim _{n \rightarrow \infty} T^{n} v=z$ and $T z=z$. On the contrary, suppose that $x \neq z$. Letting $n \rightarrow \infty \begin{gathered}n \rightarrow \infty \\ \text { trong (3.39), we have }\end{gathered}$

$$
\frac{1}{s} d(x, z) \leq \liminf _{n \rightarrow \infty} C_{s}\left(x_{n}, x_{n+1}\right) \leq \limsup _{n \rightarrow \infty} C_{s}\left(x_{n}, x_{n+1}\right)=\operatorname{sd}(x, z) .
$$

Letting $n \rightarrow \infty$ in (3.38) and using (3.40), we have

$$
d(x, z)=\frac{1}{s} s d(x, z) \leq \limsup _{n \rightarrow \infty} \beta\left(C_{s}\left(x_{n}, x_{n+1}\right)\right) s d(x, z) .
$$

This implies that $\limsup _{n \rightarrow \infty} \beta\left(C_{s}\left(x_{n}, x_{n+1}\right)\right) \geq \frac{1}{s}$. Since $\beta \in \mathcal{F}_{s}$, we have

$$
\limsup _{n \rightarrow \infty} \beta\left(C_{s}\left(x_{n}, x_{n+1}\right)\right) \leq \frac{1}{s}
$$


Therefore, we conclude that

$$
\limsup _{n \rightarrow \infty} \beta\left(C_{s}\left(x_{n}, x_{n+1}\right)\right)=\frac{1}{s}
$$

Similarly, we also see that

$$
\liminf _{n \rightarrow \infty} \beta\left(C_{s}\left(x_{n}, x_{n+1}\right)\right)=\frac{1}{s}
$$

Combining (3.41) and (3.42) gives $\lim _{n \rightarrow \infty} \beta\left(C_{s}\left(x_{n}, x_{n+1}\right)\right)=\frac{1}{s}$. Since $\beta \in \mathcal{F}_{s}$, it follows that $\lim _{n \rightarrow \infty} C_{s}\left(x_{n}, x_{n+1}\right)=0$, which contradicts (3.40). It implies that $x=z$ and hence $\lim _{n \rightarrow \infty} T^{n} v=x$.

Similarly, we also see that $\lim _{n \rightarrow \infty} T^{n} v=y$. Then, we conclude that $x=y$ and hence $T$ has a unique fixed point.

Since each metric is a $b$-metric with $s=1$, from Theorem 3.1, Theorem 3.2 and Theorem 3.3, we get following corollaries. These results are generalizations of [26, Theorem 4, Theorem 5 and Remark 9].

Corollary 3.1. Let $(X, d)$ be a metric space, $\alpha: X \times X \longrightarrow \mathbb{R}$ be a function and $T: X \longrightarrow X$ be a mapping such that

1. There exists $\beta \in \mathcal{F}$ such that for all $x, y \in X$,

$$
\alpha(x, y) d(T x, T y) \leqslant \beta(C(x, y)) C(x, y)
$$

where

$$
\begin{aligned}
C(x, y)= & \max \left\{d(x, y), d(x, T x), d(y, T y), \frac{d(x, T y)+d(y, T x)}{2},\right. \\
& \left.\frac{d\left(T^{2} x, x\right)+d\left(T^{2} x, T y\right)}{2}, d\left(T^{2} x, T x\right), d\left(T^{2} x, T y\right), d\left(T^{2} x, y\right)\right\} .
\end{aligned}
$$

2. $T$ is a triangular $\alpha$-orbital admissible.

3. There exists $x_{1} \in X$ such that $\alpha\left(x_{1}, T x_{1}\right) \geqslant 1$.

4. $T$ is continuous.

Then $T$ has a fixed point $z \in X$ and $\left\{T^{n} x_{1}\right\}$ converges $z$.

Corollary 3.2. Let $(X, d)$ be a metric space, $\alpha: X \times X \longrightarrow \mathbb{R}$ be a function and $T: X \longrightarrow X$ be a mapping such that 
1. There exists $\beta \in \mathcal{F}$ such that for all $x, y \in X$,

$$
\alpha(x, y) d(T x, T y) \leqslant \beta(C(x, y)) C(x, y)
$$

where

$$
\begin{aligned}
C(x, y)= & \max \left\{d(x, y), d(x, T x), d(y, T y), \frac{d(x, T y)+d(y, T x)}{2},\right. \\
& \left.\frac{d\left(T^{2} x, x\right)+d\left(T^{2} x, T y\right)}{2}, d\left(T^{2} x, T x\right), d\left(T^{2} x, T y\right), d\left(T^{2} x, y\right)\right\} .
\end{aligned}
$$

2. $T$ is a triangular $\alpha$-orbital admissible.

3. There exists $x_{1} \in X$ such that $\alpha\left(x_{1}, T x_{1}\right) \geqslant 1$.

4. If $\left\{x_{n}\right\}$ is a sequence in $X$ such that $\alpha\left(x_{n}, x_{n+1}\right) \geqslant 1$ for all $n \geqslant 1$ and $\lim _{n \rightarrow \infty} x_{n}=x \in X$, then there exists a subsequence $\left\{x_{n(k)}\right\}$ of $\left\{x_{n}\right\}$ such that $\alpha\left(x_{n(k)}, x\right) \geqslant 1$ for all $k \geqslant 1$.

Then $T$ has a fixed point $z \in X$ and $\left\{T^{n} x_{1}\right\}$ converges $z$.

Corollary 3.3. Let $(X, d)$ be a metric space, $\alpha: X \times X \longrightarrow \mathbb{R}$ be a function and $T: X \longrightarrow X$ be a mapping such that

1. All the hypotheses $s$ in Corollary 3.1 or Corollary 3.2 are satisfied.

2. If $x \neq y$ are two fixed points of $T$, then there exists $v \in X$ such that

$$
\alpha(x, v) \geq 1, \alpha(y, v) \geq 1 \text { and } \alpha(v, T v) \geq 1 \text {. }
$$

Then $T$ has a unique fixed point.

Remark 3.1. Theorem 3.1, Theorem 3.2 and Theorem 3.3 are generalizations of [14, Theorem 3.8].

Finally, we give some examples to support our results. The following example is an illustration of Theorem 3.2. This example also proves that Theorem 3.2 is a proper generalization of [14, Theorem 3.8].

Example 3.1. Let $X=[-2,-1] \cup\{0\} \cup[1,2]$ and $b$-metric $d$ be defined by $d(x, y)=$ $(x-y)^{2}$ for all $x, y \in X$. Then $(X, d, s)$ is a $b$-metric space with $s=2$. Let $T: X \longrightarrow X$ be defined by

$$
T x= \begin{cases}-x & \text { if } x \in[-2,-1) \cup(1,2] \\ 0 & \text { if } x \in\{-1,0,1\} .\end{cases}
$$

By choosing $x=1, y=2$, we have $d(x, y)=d(1,2)=1$ and $d(T x, T y)=d(0,-2)=4$. Then, if the condition $d(T 1, T 2) \leqslant \beta(d(1,2)) d(1,2)$ is satisfied, then $4 \leq \beta(1)$. This 
contradicts $\beta \in \mathcal{F}_{s}$. Therefore, [14, Theorem 3.8] cannot be used to prove the existence of a fixed point of $T$.

Define a mapping $\alpha: X \times X \longrightarrow \mathbb{R}$ by

$$
\alpha(x, y)= \begin{cases}1 & \text { if } x, y \in\{-1,0,1\} \\ \frac{1}{6} & \text { if } x y>0 \\ 0 & \text { otherwise }\end{cases}
$$

We claim that $T$ is triangular $\alpha$-orbital admissible mapping. Indeed, for $x \in X$ such that $\alpha(x, T x) \geqslant 1$, it implies that $x \in\{-1,0,1\}$. Then $\alpha\left(T x, T^{2} x\right)=\alpha(0,0)=1$. It implies that $T$ is $\alpha$-orbital admissible. For $x, y \in X$ such that $\alpha(x, y) \geq 1$ and $\alpha(y, T y) \geq 1$, it follows that $T y=0$ and $x \in\{-1,0,1\}$. This implies that $\alpha(x, T y) \geq 1$. Therefore, $T$ is a triangular $\alpha$-orbital admissible mapping.

Let $\left\{x_{n}\right\} \subset X$ such that $\alpha\left(x_{n+1}, x_{n}\right) \geq 1$ and $\lim _{n \rightarrow \infty} x_{n}=x \in X$. We conclude that $x, x_{n} \in\{-1,0,1\}$ and hence $\alpha\left(x_{n}, x\right) \geq 1$.

Finally, we claim that $T$ is a generalized $\alpha$-Geraghty contraction type mapping with $\beta(t)=\frac{1}{3}$ for all $t \geq 0$. Indeed, for all $x, y \in X$, we consider the following cases.

Case 1. $x, y \in[-2,-1)$. Then $d(T x, T y)=d(-x,-y)=(x-y)^{2} \leq 1$ and $C_{s}(x, y) \geq$ 4. This implies that

$$
s \alpha(x, y) d(T x, T y) \leq 2 \times \frac{1}{6} \times 1=\frac{1}{3} \text { and } \beta\left(C_{s}(x, y)\right) C_{s}(x, y) \geq \frac{1}{3} \times 4=\frac{4}{3} .
$$

Case 2. $x, y \in(1,2]$. Then $d(T x, T y)=d(-x,-y)=(x-y)^{2} \leq 1$ and $C_{s}(x, y) \geq 4$. This implies that $s \alpha(x, y) d(T x, T y) \leq 2 \times \frac{1}{6} \times 1=\frac{1}{3}$ and $\beta\left(C_{s}(x, y)\right) C_{s}(x, y) \geq \frac{1}{3} \times 4=\frac{4}{3}$.

Case 3. $x \in[-2,-1), y=-1$ or $x \in(1,2], y=1$. Then $d(T x, T y)=d(-x, 0)=x^{2}$ and $C_{s}(x, y) \geq x^{2}$. This implies that

$$
s \alpha(x, y) d(T x, T y) \leq 2 \times \frac{1}{6} x^{2}=\frac{x^{2}}{3} \text { and } \beta\left(C_{s}(x, y)\right) C_{s}(x, y) \geq \frac{x^{2}}{3} .
$$

Case 4.: $x \in[-2,-1) \cup(1,2], y=0$. Then $d(T x, T y)=d(-x, 0)=x^{2}$ and $C_{s}(x, y) \geq x^{2}$. This implies that

$$
s \alpha(x, y) d(T x, T y) \leq 2 \times 0 \times x^{2}=0 \text { and } \beta\left(C_{s}(x, y)\right) C_{s}(x, y) \geq \frac{x^{2}}{3}
$$

Case 5. $x, y \in\{-1,0,1\}$. Then $d(T x, T y)=d(0,0)=0$ and $C_{s}(x, y) \geq 0$.

Case 6. $x=-1, y \in[-2,-1)$. Then $d(T x, T y)=d(0,-y)=y^{2}$ and $C(x, y) \geq 4 y^{2}$. This implies that $s \alpha(x, y) d(T x, T y) \leq 2 \times \frac{1}{6} y^{2}=\frac{y^{2}}{3}$ and $\beta\left(C_{s}(x, y)\right) C_{s}(x, y) \geq \frac{4 y^{2}}{3}$.

Case 7. $x=1, y \in(1,2]$. Then $d(T x, T y)=d(0,-y)=y^{2}$ and $C_{s}(x, y) \geq 4 y^{2}$. This implies that $s \alpha(x, y) d(T x, T y) \leq 2 \times \frac{1}{6} \times y^{2}=\frac{y^{2}}{3}$ and $\beta\left(C_{s}(x, y)\right) C_{s}(x, y) \geq \frac{1}{3} \times 4 y^{2}=\frac{4 y^{2}}{3}$.

Case 8. $x=0, y \in[-2,-1) \cup(1,2]$. Then $d(T x, T y)=d(0,-y)=y^{2}$ and $C_{s}(x, y) \geq 4 y^{2}$. This implies that $s \alpha(x, y) d(T x, T y) \leq 2 \times 0 \times y^{2}=0$ and $\beta\left(C_{s}(x, y)\right) C_{s}(x, y) \geq \frac{4 y^{2}}{3}$. 
Case 9. $x \in[-2,-1), y \in(1,2]$ or $x \in(1,2], y \in[-2,-1)$ or $x \in[-2,-1), y=0$ or $x \in(1,2], y=-1$ or $x=-1, y \in(1,2]$ or $x=1, y \in[-2,-1)$. Then $C_{s}(x, y) \geq 0$ and $s \alpha(x, y) d(T x, T y)=s \times 0 \times d(T x, T y)=0$.

By the above cases, we conclude that $s \alpha(x, y) d(T x, T y) \leq \beta(C(x, y)) C(x, y)$ and hence $T$ is a generalized $\alpha$-Geraghty contraction type mapping. Therefore, all the hypotheses in Theorem 3.2 are satisfied. So, Theorem 3.2 is applicable to $T, \alpha, \beta$ and $(X, d, s)$.

The following example proves that Corollary 3.1 is a proper generalization of [26, Theorem 4, Theorem 5].

Example 3.2. Let $X=\{1,2,3,4,5\}$ and metric $d$ be defined by

$$
d(x, y)= \begin{cases}0 & \text { if } x=y \\ 2 & \text { if }(x, y) \in\{(1,4),(4,1),(1,5),(5,1)\} \\ 1 & \text { otherwise }\end{cases}
$$

Define a map $\alpha: X \times X \rightarrow \mathbb{R}$ by

$$
\alpha(x, y)= \begin{cases}1 & \text { if }(x, y) \in\{(1,1),(1,2),(1,5),(2,1),(2,2),(2,5) \\ & (3,5),(4,5),(5,4)\} \\ 0 & \text { otherwise }\end{cases}
$$

Let $T: X \longrightarrow X$ be defined by $T 1=T 2=T 3=T 4=1, T 5=2$. Then $T$ is triangular $\alpha$-orbital admissible. Indeed, for $x \in X$ such that $\alpha(x, T x) \geqslant 1$, we conclude that $x \in\{1,2\}$. Therefore, $\alpha\left(T 1, T^{2} 1\right)=\alpha(1,1)=1$. This implies that $T$ is $\alpha$-orbital admissible. For $x, y \in X$ such that $\alpha(x, y) \geqslant 1$ and $\alpha(y, T y) \geqslant 1$, we have $x, y \in\{1,2\}$. This implies that $\alpha(x, T y)=\alpha(1,1)=1$. Therefore, we conclude that $T$ is triangular $\alpha$-orbital admissible.

By choosing $x=2, y=5$, we have $\alpha(2,5)=1, d(T x, T y)=d(1,2)=1$ and

$$
M_{T}(2,5)=\max \left\{d(2,5), d(2,1), d(5,2), \frac{d(2,2)+d(5,1)}{2}\right\}=1 .
$$

If hypothesis (1) in [26, Theorem 4, Theorem 5] is satisfied, then it implies that $\beta(1) \geq 1$. This contradicts $\beta \in \mathcal{F}$. Thus, $[26$, Theorem 4, Theorem 5] is not applicable to $T,(X, d)$ and $\alpha$.

Let $\beta(t)=\frac{2}{3}$ for all $t \geq 0$. For $x, y \in X$, put

$$
L=\alpha(x, y) d(T x, T y), R=\beta(C(x, y)) C(x, y)=\frac{2}{3} C(x, y)
$$

Then we have the following table. 


\begin{tabular}{|c|c|c|c|c|c|c|}
\hline$x$ & $y$ & $\alpha(x, y)$ & $d(T x, T y)$ & $L$ & $C(x, y)$ & $R$ \\
\hline 1 & 1 & 1 & 0 & 0 & 0 & 0 \\
\hline 1 & 2 & 1 & 0 & 0 & 1 & $\frac{2}{3}$ \\
\hline 1 & 3 & 0 & 0 & 0 & 1 & $\frac{2}{3}$ \\
\hline 1 & 4 & 0 & 0 & 0 & 2 & $\frac{4}{3}$ \\
\hline 1 & 5 & 1 & 1 & 1 & 2 & $\frac{4}{3}$ \\
\hline 2 & 1 & 1 & 0 & 0 & 1 & $\frac{2}{3}$ \\
\hline 2 & 2 & 1 & 0 & 0 & 1 & $\frac{2}{3}$ \\
\hline 2 & 3 & 0 & 0 & 0 & 1 & $\frac{2}{3}$ \\
\hline 2 & 4 & 0 & 0 & 0 & 2 & $\frac{4}{3}$ \\
\hline 2 & 5 & 1 & 1 & 1 & 2 & $\frac{4}{3}$ \\
\hline 3 & 1 & 0 & 0 & 0 & 1 & $\frac{2}{3}$ \\
\hline 3 & 2 & 0 & 0 & 0 & 1 & $\frac{2}{3}$ \\
\hline 3 & 3 & 0 & 0 & 0 & 1 & $\frac{2}{3}$ \\
\hline 3 & 4 & 0 & 0 & 0 & 2 & $\frac{4}{3}$ \\
\hline 3 & 5 & 1 & 1 & 1 & 2 & $\frac{4}{3}$ \\
\hline 4 & 1 & 0 & 0 & 0 & 2 & $\frac{4}{3}$ \\
\hline 4 & 2 & 0 & 0 & 0 & 2 & $\frac{4}{3}$ \\
\hline 4 & 3 & 0 & 0 & 0 & 2 & $\frac{4}{3}$ \\
\hline 4 & 4 & 0 & 0 & 0 & 2 & $\frac{4}{3}$ \\
\hline 4 & 5 & 1 & 1 & 1 & 2 & $\frac{4}{3}$ \\
\hline 5 & 1 & 0 & 1 & 0 & 2 & $\frac{4}{3}$ \\
\hline 5 & 2 & 0 & 1 & 0 & 1 & $\frac{2}{3}$ \\
\hline 5 & 3 & 0 & 1 & 0 & $\frac{3}{2}$ & 1 \\
\hline 5 & 4 & 1 & 1 & 1 & 2 & $\frac{4}{3}$ \\
\hline 5 & 5 & 0 & 0 & 0 & 2 & $\frac{4}{3}$ \\
\hline
\end{tabular}

This implies that hypothesis (1) in Corollary 3.1 is satisfied. Therefore, all the hypotheses in Corollary 3.1 are satisfied and hence Corollary 3.1 is applicable to $T,(X, d), \alpha$ and $\beta$.

Finally, we apply Theorem 3.2 to study the existence of solutions to the nonlinear integral equation.

Example 3.3. Let $C[a, b]$ be the set of all continuous functions on $[a, b], b$-metric $d$ with $s=2^{p-1}$ defined by

$$
d(u, v)=\sup _{t \in[a, b]}|u(t)-v(t)|^{p}
$$

for all $u, v \in C[a, b]$ and some $p>1$. Consider the nonlinear integral equation

$$
u(t)=g(t)+\int_{a}^{b} K(t, x, u(x)) d x,
$$

where $t \in[a, b], g:[a, b] \longrightarrow \mathbb{R}, K:[a, b] \times[a, b] \times u[a, b] \longrightarrow \mathbb{R}$ for each $u \in C[a, b]$. Suppose that the following statements hold.

1. $g$ is continuous on $[a, b]$ and $K(t, x, u(x))$ is integral with respect to $x$ on $[a, b]$.

2. $T u \in C[a, b]$ for all $u \in[a, b]$, where $T u(t)=g(t)+\int_{a}^{b} K(t, x, u(x)) d x$ for all $t \in[a, b]$. 
3. For all $u \in C[a, b]$ and $u(x) \geq 0$ for all $x \in[a, b]$, we have $T^{2} u(x) \geq 0$ for all $x \in[a, b]$.

4. For all $x, t \in[a, b]$ and $u, v \in C[a, b]$ such that $u(x), v(x) \in[0, \infty)$ for all $x \in[a, b]$, we have

$$
\begin{aligned}
& |K(t, x, u(x))-K(t, x, v(x))| \\
\leq & \xi(t, x) \max \{|u(x)-v(x)|,|u(x)-T u(x)|,|v(x)-T v(x)|, \\
& \frac{|u(x)-T v(x)|+|v(x)-T u(x)|}{2^{p}}, \frac{\left|T^{2} u(x)-u(x)\right|+\left|T^{2} u(x)-T v(x)\right|}{2^{p}}, \\
& \left.\left|T^{2} u(x)-T u(x)\right|,\left|T^{2} u(x)-T v(x)\right|,\left|T^{2} u(x)-v(x)\right|\right\},
\end{aligned}
$$

where $\xi:[a, b] \times[a, b] \longrightarrow \mathbb{R}$ is continuous function satisfying

$$
\sup _{t \in[a, b]}\left(\int_{a}^{b} \xi^{p}(t, x) d x\right)<\frac{1}{2^{2 p-2}(b-a)^{p-1}}
$$

5. There exists $u_{1} \in C[a, b]$ such that $u_{1}(t) \geq 0$ and $T u_{1}(t) \geq 0$ for all $t \in[a, b]$.

Then nonlinear integral equation (3.43) has a unique solution in $C[a, b]$.

Proof. Define a mapping $T: C[a, b] \longrightarrow C[a, b]$ by

$$
T u(t)=g(t)+\int_{a}^{b} K(t, x, u(x)) d x
$$

for all $u \in C[a, b]$ and for all $t \in[a, b]$. It follows from hypothesis (1) and hypothesis (2) that $T$ is well-defined. Notice that the existence of a solution to (3.43) is equivalent to the existence of a fixed point of $T$. Now, we will show that all the hypotheses of Theorem 3.2 are satisfied.

Define a mapping $\alpha: C[a, b] \times C[a, b] \longrightarrow \mathbb{R}$ by

$$
\alpha(u, v)=\left\{\begin{array}{cc}
1 & \text { if } u(x), v(x) \in[0, \infty) \text { for all } x \in[a, b] \\
0 & \text { otherwise }
\end{array}\right.
$$

(1). We claim that $T$ is a generalized $\alpha$-Geraghty contraction type mapping. Indeed, let $q>1$ such that $\frac{1}{p}+\frac{1}{q}=1$. From condition (4), for all $u, v \in C[a, b]$ 
such that $u(x), v(x) \in[0, \infty)$ for all $x \in[a, b]$, we have

$$
\begin{aligned}
& 2^{p-1} \alpha(u, v)|T u(x)-T v(x)|^{p} \\
& =2^{p-1}|T u(x)-T v(x)|^{p} \\
& \leq 2^{p-1}\left|\int_{a}^{b} K(t, x, u(x)) d x-\int_{a}^{b} K(t, x, v(x)) d x\right|^{p} \\
& \leq 2^{p-1}\left|\int_{a}^{b}(K(t, x, u(x))-K(t, x, v(x))) d x\right|^{p} \\
& \leq 2^{p-1}\left(\int_{a}^{b}|K(t, x, u(x))-K(t, x, v(x))| d x\right)^{p} \\
& \leq\left[2^{p-1}\left(\int_{a}^{b} d x\right)^{\frac{1}{q}}\left(\int_{a}^{b}|K(t, x, u(x))-K(t, x, v(x))|^{p} d x\right)^{\frac{1}{p}}\right]^{p} \\
& \leq 2^{p-1}(b-a)^{p-1}\left(\int_{a}^{b} \xi^{p}(t, x) d x\right) \max \{|u(x)-v(x)|,|u(x)-T u(x)|, \\
& |v(x)-T v(x)|, \frac{|u(x)-T v(x)|+|v(x)-T u(x)|}{2^{p}}, \\
& \frac{\left|T^{2} u(x)-u(x)\right|+\left|T^{2} u(x)-T v(x)\right|}{2^{p}},\left|T^{2} u(x)-T u(x)\right|, \\
& \left.\left|T^{2} u(x)-T v(x)\right|,\left|T^{2} u(x)-v(x)\right|\right\} \\
& =2^{p-1}(b-a)^{p-1}\left(\int_{a}^{b} \xi^{p}(t, x) d x\right) C_{s}(u, v) \\
& \leq 2^{p-1}(b-a)^{p-1} \sup _{t \in[a, b]}\left(\int_{a}^{b} \xi^{p}(t, x) d x\right) C_{s}(u, v) \\
& =\lambda C_{s}(u, v)
\end{aligned}
$$

where $\lambda=2^{p-1}(b-a)^{p-1} \sup _{t \in[a, b]}\left(\int_{a}^{b} \xi^{p}(t, x) d x\right)<\frac{1}{2^{p-1}}$. This implies that condition (3.1) is satisfied with $\beta(t)=\lambda$ for all $t \geq 0$. Therefore, $T$ is a generalized $\alpha$-Geraghty contraction type mapping.

(2). We shall show that $T$ is triangular $\alpha$-orbital admissible mapping. Indeed, for $u \in C[a, b]$ such that $\alpha(u, T u) \geq 1$, we have $u(x) \geq 0$ for all $x \in[a, b]$. It follows from condition (3) that $T^{2} u(x) \geq 0$. Therefore, $\alpha\left(u, T^{2} u\right) \geq 1$ and hence $T$ is $\alpha$-orbital admissible mapping. In addition, for $u, v \in C[a, b]$ such that $\alpha(u, v) \geq 1$ and $\alpha(v, T v) \geq 1$, we have $u(x), v(x), T v(x) \geq 0$ for all $x \in[a, b]$. It implies that $\alpha(u, T v) \geq 1$. Thus, $T$ is triangular $\alpha$-orbital admissible mapping.

(3). By hypothesis $(5)$, we have $\alpha\left(u_{1}, T u_{1}\right) \geq 1$.

(4). Let $\left\{u_{n}\right\} \subset C[a, b]$ such that $\alpha\left(u_{n}, u_{n+1}\right) \geq 1$ and $\lim _{n \rightarrow \infty} u_{n}=u \in C[a, b]$. 
Then $u(x), u_{n}(x) \in[0, \infty)$ for all $x \in[a, b]$ and $n \geq 0$. Therefore, $\alpha\left(u_{n}, u\right) \geq 1$ for all $n \geq 1$.

Therefore, we conclude that all the hypotheses in 3.2 are satisfied. Thus, $T$ has a fixed point $u \in C[a, b]$ and hence equation (3.43) has a solution $u \in C[a, b]$.

The following example guarantees the existence of the functions $K$ and $g$ satisfying all the hypotheses in Example 3.3.

Example 3.4. Let $C[0,1]$ be the set of all continuous functions on $[0,1], b$-metric $d$ with $s=2$ defined by

$$
d(u, v)=\sup _{t \in[0,1]}|u(t)-v(t)|^{2}
$$

for all $u, v \in C[0,1]$. Consider the nonlinear integral equation

$$
u(t)=-\frac{t^{2}}{2 \sqrt{14}}+t+\int_{0}^{1} \frac{(x+1) t^{2} u(x)}{2 \sqrt{14}(1+u(x))} d x
$$

for all $t \in[0,1]$ and $u \in C[0,1]$. Put $g(t)=-\frac{t^{2}}{2 \sqrt{14}}+t$ and $K(t, x, u(x))=\frac{(x+1) t^{2} u(x)}{2 \sqrt{14}(1+u(x))}$ for all $x, t \in[0,1]$ and $u \in C[0,1]$. Then

(1). $g$ is continuous on $[0,1]$. Since $u \in C[0,1], K(t, x, u(x))$ is integral with respect to $x$ on $[0,1]$.

(2). For all $x, t \in[0,1]$ and the sequence $t_{n} \in[0,1]$ with $\lim _{n \rightarrow \infty} t_{n}=t$. We have

$$
\begin{aligned}
\left|T u\left(t_{n}\right)-T u(t)\right| & \leq\left|g\left(t_{n}\right)-g(t)\right|+\frac{1}{2 \sqrt{14}} \int_{0}^{1}(1+x)\left|t_{n}^{2}-t^{2}\right|\left|\frac{u(x)}{1+u(x)}\right| d x \\
& \leq\left|g\left(t_{n}\right)-g(t)\right|+\frac{1}{2 \sqrt{14}} \int_{0}^{1}(1+x)\left|t_{n}^{2}-t^{2}\right| d x \\
& =\left|g\left(t_{n}\right)-g(t)\right|+\frac{3}{\sqrt{224}}\left|t_{n}^{2}-t^{2}\right| .
\end{aligned}
$$

This implies that $T u \in C[0,1]$ for all $u \in C[0,1]$.

(3). It is easy to see that $g(t) \geq 0$ for $t \in[0,1]$. In addition, for $u \in C[0,1]$ such that $u(x) \geq 0$ for all $x \in[0,1]$, we have $K(t, x, u(x)) \geq 0$ for all $x, t \in[0,1]$. It implies that $T u(t) \geq 0$ for all $u \in C[0,1]$ and $t \in[0,1]$. This implies that for all $t \in[0,1]$ and $u \in C[0,1]$,

$$
T^{2} u(x)=g(t)+\int_{0}^{1} K(t, x, T u(x)) d x \geq 0 .
$$

(4). Let $u, v \in C[0,1]$ and $u(x), v(x) \in[0, \infty)$ for all $x \in[0,1]$, we have

$$
\begin{aligned}
|K(t, x, u(x))-K(t, x, v(x))| & =\frac{(1+x) t^{2}}{2 \sqrt{14}}\left|\frac{u(x)}{1+u(x)}-\frac{v(x)}{1+v(x)}\right| \\
& =\frac{(1+x) t^{2}}{2 \sqrt{14}}\left|\frac{u(x)-v(x)}{[1+u(x)][1+v(x)]}\right| \\
& \leq \frac{(1+x) t^{2}}{2 \sqrt{14}}|u(x)-v(x)| .
\end{aligned}
$$


By choosing $\xi(t, x)=\frac{(x+1) t^{2}}{2 \sqrt{14}}$, it see that $\xi$ is continuous, $\sup _{t \in[0,1]}\left(\int_{0}^{1} \xi^{2}(t, x) d x\right)<\frac{1}{4}$, $0 \leq|K(t, x, u(x))-K(t, x, v(x))| \leq \xi(t, x)|u(x)-v(x)|$, and hence

$$
\begin{aligned}
& |K(t, x, u(x))-K(t, x, v(x))| \\
\leq & \xi(t, x) \max \{|u(x)-v(x)|,|u(x)-T u(x)|,|v(x)-T v(x)|, \\
& \frac{|u(x)-T v(x)|+|v(x)-T u(x)|}{2^{p}}, \frac{\left|T^{2} u(x)-u(x)\right|+\left|T^{2} u(x)-T v(x)\right|}{2^{p}}, \\
& \left.\left|T^{2} u(x)-T u(x)\right|,\left|T^{2} u(x)-T v(x)\right|,\left|T^{2} u(x)-v(x)\right|\right\} .
\end{aligned}
$$

(5). By choosing $u_{1}(t)=t$ for all $t \in[0,1]$, we have $T u_{1}(t)=t$ for all $t \in[0,1]$. Then $u_{1}(t) \geq 0$ and $T u_{1}(t) \geq 0$ for all $t \in[0,1]$. It implies that $\alpha\left(u_{1}, T u_{1}\right) \geq 1$.

From the above, all the hypotheses to $K$ and $g$ in Example 3.3 are satisfied.

Acknowledgements: The authors sincerely thank three anonymous referees for their remarkable comments that helped us to improve the paper. Also, we sincerely thank Dong Thap Group of Mathematical Analysis and Applications for the discussion on this article.

\section{REF E R E N C E S}

1. A. Aghajani, M. Abbas and J. R. Roshan: Common fixed point of generalized weak contractive mappings in partially ordered b-metric spaces. Math. Slovaca. 64 (2014), no. 4, $941-960$.

2. M. Arshad, E. Ameer and E. Karapnar: Generalized contractions with triangular $\alpha$-orbital admissible mapping on Branciari metric spaces. J. Inequal. Appl. 2016:63 (2016), 1 - 21.

3. A. Aghajani And R. Arab: Fixed points of $(\psi, \phi, \theta)$-contractive mappings in partially ordered b-metric spaces and application to quadratic integral equations. Fixed Point Theory Appl. 2013:245 (2013), 1 - 20.

4. A. Amini-Harandi And H. Emami: A fixed point theorem for contraction type maps in partially ordered metric paces and applications to ordinary differential equations. Nonlinear Anal. 72 (2010), 2238 - 2242.

5. T. V. An, N. V. Dung, Z. Kadelburg and S. Radenović: Various generalizations of metric spaces and fixed point theorems. Rev. R. Acad. Cienc. Exactas Fis. Nat. Ser. A Mat. RACSAM. 109 (2014), no. 1, 175 - 198.

6. T. V. An, L. Q. TuYen and N. V. Dung: Stone-type theorem on b-metric spaces and applications. Topology Appl. 185 (2015), 50 - 64.

7. I. A. BAKHtin: The contraction principle in quasimetric spaces. Func. An., Ulianowsk, Gos. Fed. Ins. 30 (1989), $26-37$.

8. M. Boriceanu, M. Bota and A. Petrusel: Multivalued fractals in b-metric spaces. Cent. Eur. J. Math. 8 (2010), no. 2, 367 - 377. 
9. J. Caballero, J. Harjani and K. Sadarangani: A best proximity point theorem for Geraghty-contractions. Fixed Point Theory Appl. 2012:231 (2012), 1 9.

10. S. H. Cho, J. S. BAe and E. KARAPinar: Fixed point theorems for $\alpha$-Geraghty contraction type maps in metric spaces. Fixed Point Theory Appl. 2013:329 (2013), $1-11$.

11. L. B. ĆIRIĆ: A generalization of Banach's contraction principle. Proc. Amer. Math. Soc. 45 (1974), $267-273$.

12. S. CzerwiK: Contraction mappings in b-metric spaces. Acta Math. Univ. Ostrav. 1 (1993), $5-11$.

13. S. CzERWIK: Nonlinear set-valued contraction mappings in b-metric spaces. Atti Semin. Mat. Fis. Univ. Modena. 46 (1998), no. 2, 263 - 276.

14. D. Dukić, Z. Kadelburg and S. Radenović: Fixed points of Geraghty-type mappings in various generalized metric spaces. Abstr. Appl. Anal. 2011 (2011), $1-13$.

15. M. Geraghty: On contractive mappings. Proc. Am. Math. Soc. 40 (1973), 604 -608 .

16. M. E. Gordji, M. Ramezami, Y. J. Cho and S. Pirbavafa: A generalization of Geraghty's theorem in partially ordered metric spaces and applications to ordinary differential equations. Fixed Point Theory Appl. 2012:74 (2012), 1 - 9.

17. B. T. N. HAN AND N. T. HiEU: A fixed point theorem for generalized cyclic contractive mappings b-metric spaces. Facta Univ. Ser. Math. Inform. 31 (2016), no. $2,399-415$.

18. N. T. Hieu AND N. V. Dung: Some fixed point results for generalized rational type contraction mappings in partially ordered b-metric spaces. Facta Univ. Ser. Math. Inform. 30 (2015), no. 1, 49 - 66.

19. H. HuANG AND S. XU: Fixed point theorems of contractive mappings in cone b-metric spaces and applications. Fixed Point Theory Appl. 2012 (2012), 1 - 8.

20. N. Hussain, V. Parvaneh, J. R. Roshan and Z. Kadelburg: Fixed points of cyclic weakly $(\psi, \varphi, L, A, B)$-contractive mappings in ordered b-metric spaces with applications. Fixed Point Theory Appl. 2013:256 (2013), 1 - 18.

21. E. KARAPINAR: $\alpha-\psi$-Geraght contraction type mappings and some related fixed point results. Filomat. 28 (2014), no. 1, $37-48$.

22. P. Kumam, N. V. Dung and V. T. L. Hang: Some equivalences between cone b-metric spaces and b-metric spaces. Abstr. Appl. Anal. 2013 (2013), $1-8$.

23. P. Kumam, N. V. Dung and K. Sitthithakerngkiet: A generalization of Cirić fixed point theorems. Filomat. 29 (2015), no. 7, 1549 - 1556.

24. R. PANTA AND R. PANiCKeR: Geraghty and Ćirić type fixed point theorems in b-metric spaces. J. Nonlinear Sci. Appl. 9 (2016), no. 11, 5741 - 5755.

25. V. Parvaneh, J. R. Roshan and S. Radenović: Existence of tripled coincidence points in ordered b-metric spaces and an application to a system of integral equations. Fixed Point Theory Appl. 2013:130 (2013), 1 - 19.

26. O. Popescu: Some new fixed point theorems for $\alpha$-Geraghty contraction type maps in metric spaces. Fixed Point Theory Appl. 2014:190 (2014), 1 - 12. 
27. A. C. M. RAn And M. C. B. Reurings: A fixed point theorem in partially ordered sets and some applications to matrix equations. Proc. Amer. Math. Soc. 132 (2003), no. 5, 1435 - 1443.

28. J. R. Roshan, V. Parvaneh, S. Sedghi, N. Shobkolaei and W. Shatanawi: Common fixed points of almost generalized $(\psi, \varphi)_{s}$-contractive mappings in ordered b-metric spaces. Fixed Point Theory Appl. 2013:159 (2013), 1 - 23.

29. W. Shatanawi, A. Pitea and R. Lazović: Contraction conditions using comparison functions on b-metric spaces.Fixed Point Theory Appl. 2014:135 (2014), $1-11$.

30. T. SuZuki: A generalized Banach contraction principle that characterizes metric completenes. Proc. Amer. Math. Soc. 136 (2008), no. 5, 1861 - 1869.

Nguyen Trung Hieu

Faculty of Mathematics and Information Technology Teacher Education Dong Thap University, Cao Lanh City,

Dong Thap Province,

Vietnam

ngtrunghieu@dthu.edu.vn

Le Thi Chac

Faculty of Mathematics and Information Technology Teacher Education Dong Thap University, Cao Lanh City,

Dong Thap Province,

Vietnam

chacdhstoan11@gmail.com 